\title{
Carcinoma of the oesophagus in Transkei: treatment by intubation
}

\author{
M H COTTON, A M SAMMON \\ From the Department of Surgery, Umtata Hospital, Transkei, South Africa
}

\begin{abstract}
The management of carcinoma of the oesophagus poses formidable logistic problems in $\stackrel{+}{+}$ countries such as Transkei where the condition is common and resources are limited. Most patients present late, often with complications, and are reluctant to undergo major surgery. Two hundred and $\overrightarrow{\mathrm{N}}$ fifty consecutive patients who presented over nine months in Transkei were studied. The incidence윽 increased with age until 70 years and the disease occurred equally in men and women. The neoplasmwas predominantly squamous cell (243 patients, $97 \%$ ) and was found most often in the middle third@ of the oesophagus $(118,47 \%)$. On admission only eight of the 250 patients could take a semi-solid diet and only 21 a fluid diet. The policy where feasible was to introduce a Proctor Livingstone tube endoscopically through the dilated oesophageal stricture by a pulsion technique under light general $\vec{\bullet}$ anaesthesia. When abdominal perforation of the oesophagus seemed likely, retrograde intubation viaco a gastrotomy was performed. Sixty patients were not intubated, because the stricture was too proximal (47) or could not be dilated adequately (6), the lesion was suitable for resection (6), or the patient refused (1). Fifty one (27\%) patients died in hospital, 29 deaths being due to oesophageal perforation (including six of the 10 who were intubated retrogradely). The mean hospital stay was $4 \cdot 7 \%$ days. On discharge $64 \%$ of the intubated patients were able to take semi-solid food and a further $6 \%$ a fluid diet. Palliation by intubation was performed rapidly and the tube was well tolerated by patients. $\overrightarrow{0}$ The overall mortality was high, but this can be reduced by experience. Intubation is an acceptable,, cost effective solution where large numbers of patients present with advanced oesophageal carcinoma in circumstances where resources are severely limited.
\end{abstract}

\section{Introduction}

Carcinoma of the oesophagus is common in Transkei and accounts for $40 \%$ of all neoplasms. ${ }^{\prime}$ The incidence is rising ${ }^{2}$ and has been estimated to be 5-30 times as high as in the United Kingdom, and similar to that in the North Linxian region of China. ${ }^{3}$ The incidence varies considerably, however, within Transkei. ${ }^{4} \mathrm{Al}-$ though no cause has been clearly established, pipe smoking and beer drinking have been implicated; ${ }^{5}$ relative deficiencies of trace elements, riboflavin, and nicotinic acid substantially enhance the susceptibility of the oesophagus to carcinogens; ${ }^{6}$ and the corn fungus Fusarium moniliforme, which is most prevalent in endemic areas, promotes carcinogenesis. ' Over 300 patients with oesophageal carcinoma are referred annually to the central hospital in Umtata. Their

Address for reprint requests: Mr M H Cotton, Department of Surgery, Harare Hospital, PO Box ST14, Southerton, Harare, Zimbabwe.

Accepted 26 September 1988 management poses a formidable logistic problem. The patients usually present with advanced disease because many have cultural reservations about Western medical practice and symptoms often begin only wherg about two thirds of the oesophageal lumen is occluded. In neighbouring Natal a special unit using an aggressive approach has been established to cope with the problem. In an attempt to avoid this extrof expense we have sought a simple and effective methoof. using existing facilities. In a prospective study of $25 \%$ consecutive patients presenting with oesophageal carcinoma over nine months we evaluated the resultso of treatment by intubation alone.

\section{Patients and methods}

During the nine months January-September 1985 we studied prospectively 250 consecutive patients witbs carcinoma of the oesophagus. Patients presenting wit| dysphagia were sent for unprepared, unscreenefof barium swallow radiography. Those with evidence of neoplasia were assessed for symptoms, extent of 
disease, and level of debilitation. One investigator questioned all patients about the degree and duration of dysphagia and other symptoms. They were also asked about social habits-namely smoking and alcohol intake. Degrees of weight loss and dehydration were assessed clinically. Radiographs were examined for position, length, and axial deviation of oesophageal stricturing; fistula formation into the pleural space or bronchial tree; and concomitant lung abscess, pleural effusion, or pulmonary tuberculosis.

Intravenous fluids were given preoperatively to patients unable to ingest liquids and to those manifestly dehydrated. Blood was transfused only in patients with a haemoglobin concentration below $6 \mathrm{~g} / \mathrm{dl}$. The only contraindication to operative intervention was impaired consciousness; such patients were excluded from the study.

After thiopentone, nitrous oxide, halothane, and suxamethonium had been administered, the vocal cords were examined and endotracheal intubation was carried out. The Negus oesophagoscope was then passed for visualisation and biopsy of the stricture (benign strictures of the oesophagus were excluded from the study). Antegrade dilatation of the stricture and intubation with the modified Proctor-Livingstone tube (Latex Products, Johannesburg) was carried out as described by Hegarty et al. ${ }^{8}$ Aspiration of acid onto litmus paper via a nasogastric tube confirmed correct placement of the endooesophageal prosthesis and free passage into the stomach. Repeat dilatation was attempted after three days if the first attempt had to be abandoned. Patients with high lesions unsuitable for intubation because of resulting airway obstruction were referred for radiotherapy to East London, a three hour journey by road, after dilatation of the stricture had made adequate swallowing possible. Strictures through which a bougie could not be passed at the second attempt or where perforation into the abdomen was suspected were intubated in retrograde fashion via a small gastrotomy (traction method). ${ }^{9}$

Clear fluids were given orally after antegrade intubation when the patients were suitably awake, but were withheld for 48 hours after a gastrotomy. A fluid diet and subsequently semi-solids were given if these were tolerated. Sleep in a semi-erect position was advised. Patients were allowed home once they could swallow to their satisfaction their normal food, which was mainly maize porridge, or if they were anxious to go home even though optimal swallowing had not been achieved.

No long term follow up was possible as most patients lived a great distance from the hospital and had meagre resources for transport.

\section{Results}

The age and sex distribution of the 250 patients is illustrated in figure 1. Most patients were in the 60-69 year age group but 11 were below 40 years and three below 30 years old, two of whom were pregnant. Men and women were about equally affected. Social habits are shown in fig 2 . The popularity of the traditional long Xhosa pipe and locally made Xhosa beer is evident.

All but three patients $(98 \%)$ presented with dysphagia and could point to the site of obstruction with remarkable accuracy. Regurgitation was present in $175(70 \%)$. Retrosternal pain was uncommon, being present and made worse by swallowing in 12 cases. Other symptoms included cough (45), dyspnoea (24), backache (2), vomiting (8), lump in the throat (1), and hoarse voice (3). The degree of dysphagia is shown in

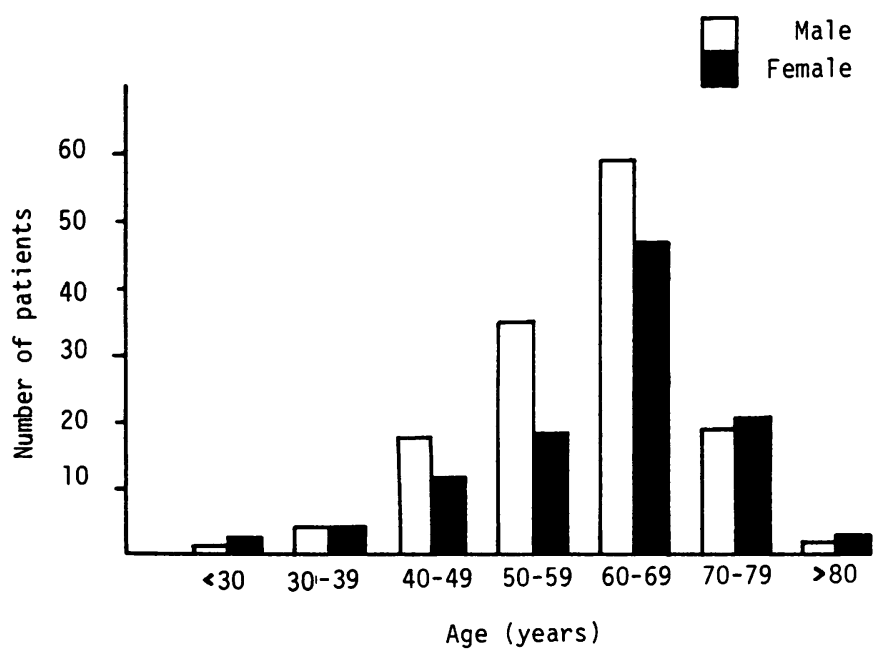

Fig 1 Age and sex distribution of the 250 patients with oesophageal cancer. 


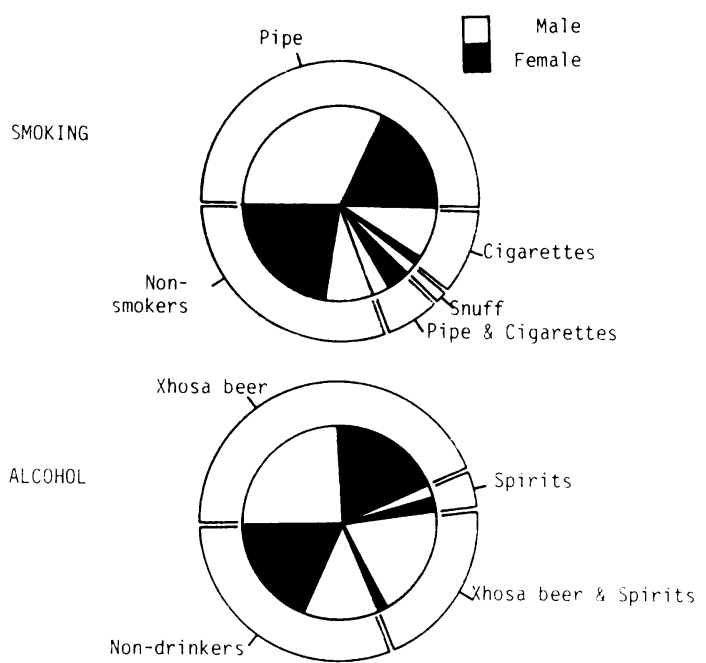

Fig 2 Social habits of the 250 patients, showing the popularity of the traditional long pipe and the local Xhosa beer.

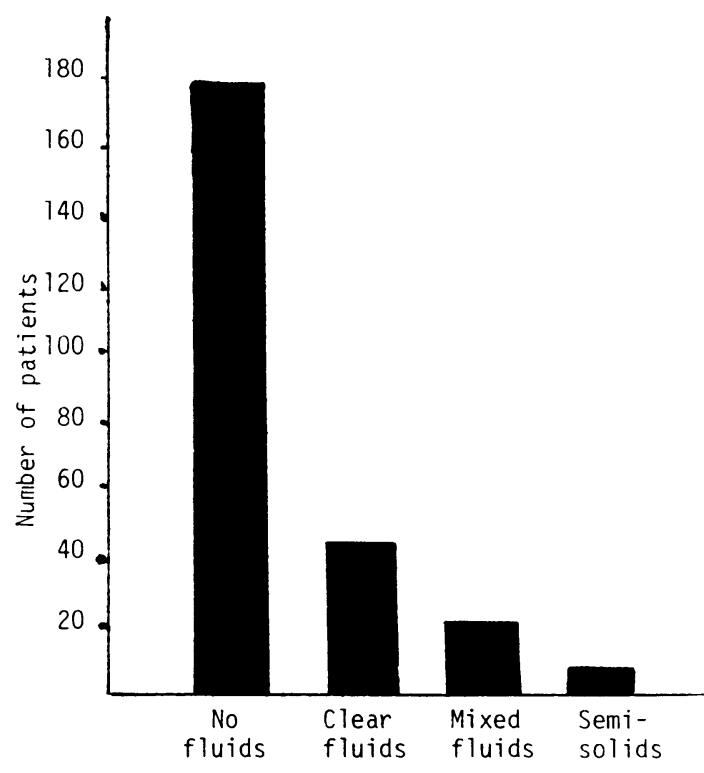

Ability to swallow

Fig 3 Degree of dysphagia at presentation.

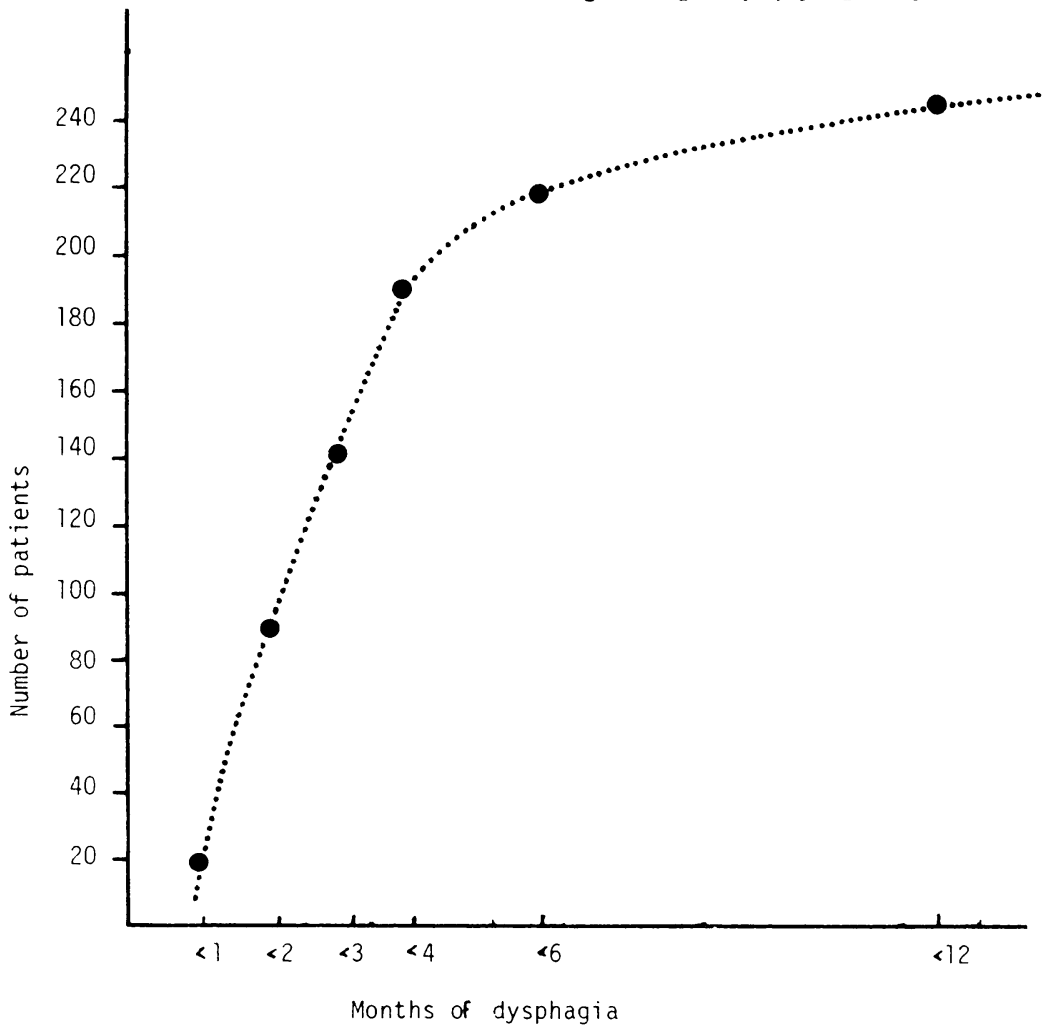

Fig 4 Duration of dysphagia. 


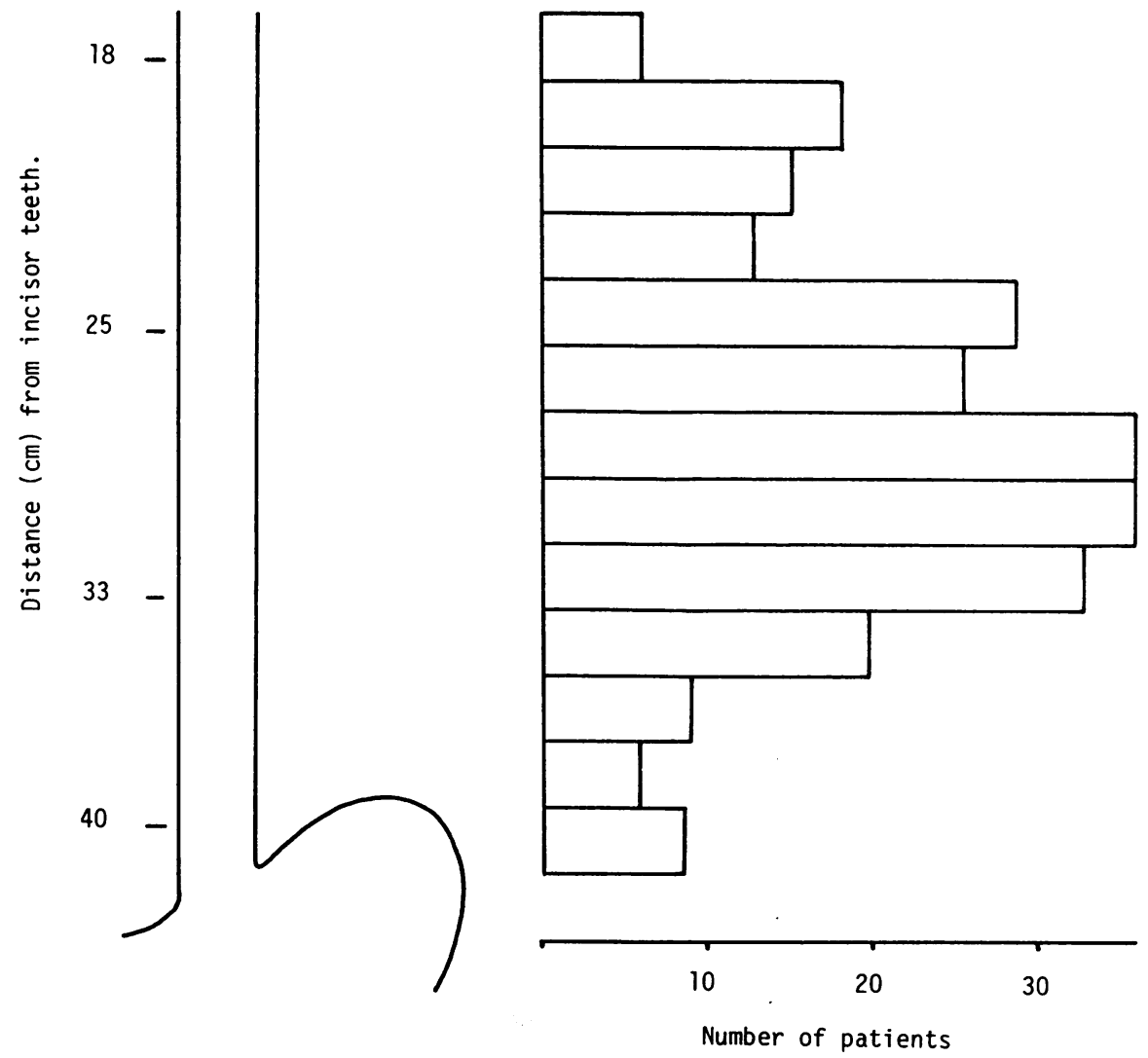

Fig 5 Distribution of malignant lesions along the oesophagus.

figure 3. Advanced disease predominated, although most patients had complained of symptoms for less than four months (fig 4). One hundred and seventy nine $(72 \%)$ had almost complete obstruction of the oesophagus.

Clinically, 158 patients (63\%) had more than $15 \%$ weight loss and $141(56 \%)$ were considerably dehydrated. Blood transfusions were given to two patients. Strictures over $6 \mathrm{~cm}$ long were found in 108 $(43 \%)$ and axial deviation in $85(34 \%)$. Tracheal spillover was evident in $98(39 \%)$, fistula formation in 15 , vocal cord palsy in six, lung abscess in four, pleural effusion in eight, and pulmonary tuberculosis (confirmed by spectrum analysis) in 18 .

Malignant stricturing of the oesophagus was confirmed histologically in all patients. The lesions were distributed throughout the oesophagus (fig 5), the greatest number being in the mid oesophagus. Histologically all but a few lesions were squamous cell carcinoma. In the upper third of the oesophagus leiomyosarcoma (1), adenocarcinoma (1), and anaplastic spindle cell tumour (1) occurred also; in the middle third anaplastic spindle cell tumour (2) and in the abdominal oesophagus adenocarcinoma (1) were found.

Dilatation of the malignant stricture was uncomplicated in 197 patients (79\%) but difficult in $47(19 \%)$. Blind dilatation was carried out in two patients, one with severe cervical spondylosis, and one where a large proximal tumour prevented passage of the oesophagoscope. Dilatation was not achieved at the first attempt in 16 cases, but was successful on the second attempt in 10; the strictures of the remaining six patients proved impassable.

One hundred and ninety intubations were performed, all but 10 by the pulsion method. Of the patients not intubated, $\mathbf{4 0}$ had a stricture that was too proximal and in a further seven intubation caused airway obstruction, necessitating removal of the tube. Of the remainder, six had strictures that could not be dilated, six had tumours suitable for resection on the basis of established criteria ${ }^{10}$ (only four of these were operated on at Umtata and two of these died in hospital), and one patient refused intubation but agreed to dilatation of his stricture.

Early discharge was favoured. Before intubation 
only seven of the 190 patients subsequently intubated could ingest semi-solid food and a further 17 mixed fluids. On discharge $121(64 \%)$ could take semi-solids (maize porridge) and their psychological relief was dramatic. Seven had to have the tube replaced, six after it had migrated distally and one because of disintegration of the tube, possibly through the action of gastric juice. Six patients had to have their tubes unblocked endoscopically, all with satisfactory results. Twelve patients could take only fluids after intubation but wished to go home nevertheless; four patients found no improvement after placement of the tube but refused further intervention, and tubes were removed from two patients at their request.

Overall mortality from intubation was high $(51 / 190$, $27 \%)$, though it was lower $(8 / 66,12 \%)$ for the surgeon who performed the greatest number of intubations. Of the 51 deaths, 29 were attributed to oesophageal perforation and the remainder to bronchopneumonia (9), respiratory obstruction (7), massive haemorrhage (1), fulminating pulmonary tuberculosis (1), and irreversible cachexia (4). Of the 15 patients with known fistulas, nine $(60 \%)$ fared well after intubation, the tube preventing spillage of oesophageal contents. Six of the patients with fistulas died, two from pneumonia and inanition, one from a mass in the neck causing respiratory obstruction, one from tumour invading the trachea, one from necrotic tumour pushed into the airway by the tube, and one from enlargement of the fistula by a bougie that the tube failed to occlude.

Of those who died, 29 had lost more than $15 \%$ of their body weight and were considerably dehydrated. Six of the 10 patients who underwent retrograde intubation died within seven days.

\section{Discussion}

Oesophageal carcinoma is the most common malignancy in Transkei. It occurs at an earlier age than in the United Kingdom and has an almost equal sex incidence. The distribution of lesions also follows a different pattern, the mid oesophagus being the most frequent site and squamous carcinomas far outnumbering adenocarcinomas.

Late presentation with advanced inanition is common. A similar pattern was reported from Uganda. ${ }^{1}$ Palliation is thus the aim of treatment in Transkei for all but the occasional patient who presents with an early lesion. No radiotherapy facilities exist in Umtata and it would not be feasible to refer all patients with oesophageal carcinoma to the nearest centre, $235 \mathrm{~km}$ away. Laser equipment is also not available.

Endoscopic intubation, however, requires simple anaesthesia, is rapid, and is a skill that is readily acquired. Swallowing is immediately restored, with great psychological impact, and removes the inevitability of aspiration. Only six of the 199 patient discharged from hospital gained no improvemen $\Phi$ from the procedure; 12 others were able to swallow only mixed fluids but were satisfied with this. The modified Proctor-Livingstone tube, which is wide an flexible and does not readily dislocate, was weth tolerated. Obstruction by food debris can be cleared by fizzy drinks, such as Coca Cola, and rarely require? endoscopy. Intubation is the treatment of choice for tracheo-oesophageal fistulas.

There was, however, a high mortality $(27 \%)$ in thi series. A similar mortality was reported from Barage wanath Hospital, Soweto ${ }^{12}$; but in the series from Durban, where a special unit was set up, mortality was only $16 \% .{ }^{13}$ A comparable rate $(12 \%)$ was recorded b\% the surgeon who performed most intubations in ouf series, suggesting that increased experience ma $\vec{y}$ reduce the frequency of oesophageal perforation which accounted for over half the deaths. The use of fibreoptic endoscope for distal lesions may reduce the mortality further, as retrograde intubation gave discouraging results. ${ }^{14}$

An average hospital stay of well under a week enabled patients to return quickly to their families reducing the hospital burden, and encouraging others with the same disease to come forward. There was however, no possibility of assessing pain levels, qualit के of life or long term sequelae of intubation because of the enormous difficulties that would be encountered i $\overrightarrow{\overrightarrow{0}}$ following these patients. Nevertheless, intubatio appears to provide an effective palliation for advanced oesophageal carcinoma in a setting where resources. are very limited.

We are indebted to $\mathrm{Mr} \mathrm{O}$ duP Fehrsen and $\mathrm{Mr}$ Mugwanya for permission to study their patients; $D_{3}$ D T McLeod for his help and encouragement in the preparation of this paper; and Dr A vd Welden for he? assistance with data collection.

\section{References}

1 Rose EF, Fellingham SA. Cancer patterns in Transkeô $S$ Afr J Sci 1981;77:555-61.

2 Rose EF, Procter DSC. The Butterworth diagnostic clinif for oesophageal cancer in Transkei: a five-year survey. S Afr Med J 1970;44:1199-203.

3 McKeown KC. Carcinoma of the oesophagus. J R Cå̈ Surg Edinb 1979;24:253-74.

4 Rose EF, McGlashan ND. The spatial distribution of oesophageal carcinoma in the Transkei, South Africad Br J Cancer 1975;31:197-206.

5 McGlashan ND, Bradshaw E, Harington JS. Carcinoma of the oesophagus and the use of tobacco and alcohol beverages in Transkei 1975-6. Int J Cancer 198\% 29:249-56. 
6 van Rensburg SJ. Recent studies on the aetiology of oesophageal cancer. $S$ Afr Cancer Bull 1985;29:22-31.

7 Marasas WFO, Wehner FC, van Rensburg SJ, van Schalkwyk DJ. Mycoflora of corn produced in human esophageal cancer areas in Transkei, Southern Africa. Phytopathology 1981;71:792-6.

8 Hegarty MM, Angorn IB, Bryer JV, Henderson BJ, Le Roux BT, Logan A. Pulsion intubation for palliation of carcinoma of the oesophagus. Br J Surg 1977; 64:160-5.

9 Celestin LR. Permanent intubation in inoperable carcinoma of the oesophagus and cardia. Ann R Coll Surg Engl 1959;25:165-70.
10 Angorn IB. Intubation in treatment of carcinoma of the oesophagus. World J Surg 1981;5:535-41.

11 Bonelli AM. Carcinoma of the oesophagus in Uganda. Proc Assoc Surg E Afr 1980;3:96-101.

12 Diamantes T, Mannell A. Oesophageal intubation for advanced oesophageal cancer: the Baragwanath experience 1977-1981. Br J Surg 1983;70:555-7.

13 Angorn IB, Hegarty MM. Palliative pulsion intubation in oesophageal carcinoma. Ann $R$ Coll Surg Engl 1979;61:212-6.

14 Ogilvie AL, Dronfield MW, Ferguson R, Atkinson M. Palliative intubation of oesophagogastric neoplasms at fibreoptic endoscopy. Gut 1982;23:1060-7. 